\title{
Clustered versus catastrophic global vertebrate declines
}

\author{
Brian Leung ${ }^{1,2^{*}}$ \\ Anna L. Hargreaves ${ }^{1}$ \\ Dan A. Greenberg ${ }^{3}$ \\ Brian McGill ${ }^{4}$ \\ Maria Dornelas ${ }^{5}$ \\ Robin Freeman ${ }^{6}$
}

${ }^{1}$ Department of Biology, McGill University, Montreal, Quebec, Canada H3A 1B1

${ }^{2}$ School of Environment, McGill University, Montreal, Quebec, Canada, H3A 2A7

${ }^{3}$ Department of Biological Sciences, Simon Fraser University, Burnaby, British Columbia, Canada V5A 1 S6

${ }^{4}$ School of Biology and Ecology and Mitchell Center for Sustainability Solutions, University of Maine, Orono, ME USA

${ }^{5}$ Centre for Biological Diversity, University of St Andrews, St Andrews KY16 $9^{\text {TH }}$, UK

${ }^{6}$ Indicators and Assessments Unit, Institute of Zoology, Zoological Society of London, London, NW1 4RY

*Corresponding author email: brian.leung2@mcgill.ca

\section{Summary}

Recent analyses have reported catastrophic global declines of vertebrate populations ${ }^{1,2}$. Yet distilling many trends into a global mean index obscures variation that can inform conservation, and can be sensitive to analytical decisions. For example, whereas earlier analyses estimated a mean vertebrate decline of $>50 \%$ since 1970 (Living Planet Index: LPI $^{2}$ ), we find that this estimate is driven by $<3 \%$ of populations; excluding these extremely declining populations switches the global trend to an increase. The sensitivity of global mean trends to outliers suggests that more informative indices are needed. We propose an alternative approach, identifying clusters of extreme decline (or increase) that differ statistically from the majority of population trends. We show that, of LPI's 57 taxonomic-geographic systems, 16 systems contain clusters of extreme decline (comprising $\sim 1 \%$ of populations, occurring disproportionately in larger animals) and 7 contain extreme increases ( $0.4 \%$ of populations). The remaining $98.6 \%$ of populations across all systems showed no mean global trend. However, when analyzed separately, three systems were declining strongly with high certainty (all Indo-Pacific), and seven were declining strongly but with less certainty (mostly reptile-amphibian groups). Accounting for extreme clusters fundamentally alters interpretation of global vertebrate trends and should be used to help prioritize conservation effort. 
Rapid global change is threatening species across the globe ${ }^{1}$. Quantifying biodiversity trends is important to assess whether current investment is slowing or reversing declines, and identify regions and taxa of concern. While distilling disparate population trends into a single global index can focus attention on biodiversity trends ${ }^{2,3,4}$, simple metrics can distort the full picture.

Estimates of global biodiversity trends vary depending on their data and mathematical model. The most apocalyptic garner extensive press coverage, even when based on controversial data (e.g., 'biological annihilation'5 using trend estimates based largely on expert opinion; 'insect Armageddon' based on data disputed by the original collectors ${ }^{6}$ ). But even analyses of the best available data reach conflicting results. Analysis of a global dataset of abundance time-series estimated that on average vertebrate populations have declined by $>50 \%$ since 1970 (Living Planet Index 2 :LPI), but other global analyses found that mean population size ${ }^{7,8}$ and species richness ${ }^{9,10}$ have remained stable over similar timeframes. Explanations for the discrepancies have been proposed ${ }^{11},{ }^{12},{ }^{, 13}$, but not resolved.

One crucial consideration is that summary indices may be easily misinterpreted. Calculating the geometric mean across populations is the most common and straightforward approach, but is strongly influenced by extremes. To illustrate, imagine an ecosystem in which one population declined by $99 \%$. Even if a second population increased 50 fold or 393 populations increased by $1 \%$ (i.e. a large net increase) a geometric mean would show a catastrophic $50 \%$ decline. Thus, a geometric mean decline of $50 \%$ could arise from significant, widespread loss is occurring across many populations (we term this the 'Catastrophic declines' hypothesis), or from a few scenarios involve important conservation issues, but suggest vastly different underlying problems and mitigation strategies ${ }^{14}$, thus distinguishing between them is of real-world importance.

We derive a Bayesian hierarchical mixture (BHM) model to distinguish between the Catastrophic and Clustered decline hypotheses. The model statistically separates population trends into extreme declines, typical trends, and extreme increases (Fig 1), while accounting for time-series size, within population fluctuations, number of populations, and among-population variance. We test declines in abundance for $>14000$ vertebrate populations $\left(\mathrm{LPP}^{21}\right)$. We chose LPI data for its impressive scope, because the data and analytical details were publicly available, and because previous analyses of these data suggested widespread, global declines $^{2}$.

We first examined whether the previous mean decline estimate of $>50 \%{ }^{2}$ was sensitive to extreme populations: robust declines would support the Catastrophe hypothesis; high sensitivity to few populations would support the Cluster hypothesis (Fig 1). We then applied our BHM model to assess the evidence for Catastrophic vs. Clustered declines globally and by region, taxonomy. Finally, we explore two additional conservation issues. First, we test whether declines occur disproportionately in larger animals (big animals tend to have lower reproductive rates), which might release small animals from predation ${ }^{18}$. Second, previous analyses often excluded time-series with few datapoints ${ }^{10,12,19}$, but small time-series make up most of available data. We test the effects of their exclusion ${ }^{20}$. 


\section{Results}

Sensitivity of Geometric Mean index to extreme populations

The geometric mean underlying the LPI analysis was highly sensitive to extreme populations. Excluding only the $2.4 \%$ most strongly declining populations (354 of 14700 populations) reversed the estimate of global vertebrate trends from $>50 \%$ loss to slightly positive growth (Fig 2). Similarly, excluding $2.4 \%$ of the most strongly increasing populations strengthened the mean decline to $71 \%$. High sensitivity suggests that extreme populations are disproportionately affecting global trend estimates, such that clusters of extreme population decline should be considered explicitly.

\section{Evidence for Clustered declines}

Among LPI's 57 domain-realm-taxon systems, 16 systems contained clusters of extreme decline, and 8 contained clusters of extreme growth (3 systems are repeated, i.e. had both clusters of extreme decline and growth; Fig 3, SI Table S2). Together, clusters of extreme decline accounted for only $1 \%$ of populations across systems ( $2 \%$ of populations in the 16 systems in which they occurred). The mean population trend for extreme declining clusters across the 16 systems was $\theta_{2}=-3.94$, or approximately $98 \%$ loss per year, and deviated substantially from the mean trend of the primary cluster in those systems. Clusters of extreme growth accounted for $0.4 \%$ of populations across systems $(2.4 \%$ in the 8 systems where they occurred), with $\theta_{2}=3.51$, ie an explosive 33x growth per year (Fig 3, SI Table S2).

Extreme clusters showed some taxonomic and geographic patterns. The largest cluster of extreme declines was in arctic marine mammals, accounting for $7.6 \%$ of populations in that system. However, mammal systems generally had the fewest clusters of extreme decline [ $19 \%$ of 16 systems], followed by reptile/amphibian systems [ $21 \%$ of 14 systems], whereas bird and fish systems had more clusters of extreme declines [31\% of 16 and $45 \%$ of 11 systems, respectively; Fig 3). Clusters of extreme decline occurred throughout the world with half occurring in marine realms, whereas extreme increases occurred more in temperate regions or terrestrial realms (Fig 3).

Extreme population trends occurred predominantly in small time-series. Excluding time-series with $<10$ points removed all but two extreme clusters, but also removed $52 \%$ of the data (see SI Table S3). The higher frequency of extreme trends among small time-series was also apparent in the raw data (Figure 4). Thus the decision of whether to include small time-series will have large effects on the resulting estimates of global trends.

Body size was related to population trends. Larger species had three times more extreme declines than increases ( 15 vs 5 clusters of extreme decline vs extreme increase). Comparatively, smaller species had half as many (8) extreme declining and disproportionately more (7) extreme increasing clusters (SI Table S4). While size-specific models included fewer populations, especially for smaller species, the number of clusters was not uniformly lower (as might be expected given a reduction in power), so the differential occurrence of declining versus increasing extreme clusters suggests large animals are more vulnerable to extreme declines. 

of populations across the 57 LPI systems. The overall growth rate of primary clusters was close to zero: $\theta_{1}=-0.00035$, corresponding to $\sim 1.7 \%$ loss over 50 years, given a constant rate across populations and time (Fig 5). Also, in contrast to extreme clusters, primary cluster trends were robust to time-series size, as excluding series with $<10$ data points yielded a similar overall global trend $\left(\theta_{1}=+0.0043\right)$ (Extended data Figure E3).

While the global BHM model reveals considerably more nuance than a geometric mean index, analyzing across systems still masked important patterns. When systems were analyzed separately (SI Table S2), primary population clusters were strongly declining $\left(\theta_{1}<-0.015\right)$ with high certainty (95\% credible intervals not overlapping zero) in three systems, all in the IndoPacific Realm (freshwater mammals, freshwater birds, terrestrial birds; red distributions, Fig 3). This suggests this region has the highest risk of system-wide decline and should be a conservation priority. In contrast, the primary cluster was increasing with high certainty in seven systems, six of which were in temperate regions. Seven additional systems had strongly declining primary population clusters but with less certainty (95\% credible intervals overlapped zero), four of which were amphibian/reptile groups. 14 systems showed strong but low-certainty increases, with no obvious taxonomic nor geographic patterns (Fig 3).

Each primary cluster also contained variation among populations. In the 10 systems with significant (red) or non-significant (orange) mean declines $\left(\theta_{1}<-0.015\right), 87 \%$ of the individual populations showed strong declines (Fig 5). These 10 systems accounted for $\sim 20 \%$ of total global vertebrate populations, but $\sim 61 \%$ of strong declines. The multimodality observed in Fig 5 , was an outcome of aggregating unimodal primary clusters across systems, and suggests heterogeneous stressor levels among systems (i.e., similar principles as those causing extreme clusters within systems). The remaining $\sim 11 \%$ of strongly declining populations were distributed across 47 of 57 systems; it is unclear whether they represent a deviation from natural dynamics expected in any naturally variable system.

Primary cluster trends were related to body size, but not as predicted. Compared to overall patterns, for larger animals the same systems showed significant declines and increases, but two additional temperate systems showed significant increases (Extended data Fig E4; SI Table S4). Smaller species also appeared to decline more than larger species; there were 27 systems where smaller species had more negative growth rates than larger species, versus 18 where the reverse was true. However, analyses of smaller species were based on a substantially fewer populations and trends were generally not significant (SI Table S4), so patterns remain 166 tentative.

\section{Discussion}

168 By re-analysing the most comprehensive dataset of global wildlife population trends available, 169 we show that previously estimated global declines are driven by a few extremely declining 170 populations. Removing only $2.4 \%$ of declining populations reversed the estimated global trends 171 from $>50 \%$ mean decline since 1970 to slightly positive growth. Our BHM model revealed that 
clusters of extreme decline are widespread and occur disproportionately in larger species, and

173 that a few clusters of extreme increase also exist and occur disproportionately in smaller species. This is consistent with previous arguments of "trophic downgrading"18.

Clusters of extreme declines were largely due to small time-series. However, neither random sampling error nor "saw tooth" population dynamics (wherein ultimately stable populations experience sudden declines followed by gradual increases) can fully explain this association (see Supplementary information for full discussion). Additional explanations are needed. Extreme trends could reflect transient populations that naturally leave or enter a survey area ${ }^{22}$, which could represent natural dynamics. Alternatively, researchers may stop sampling after populations become (close to) extirpated, although the converse has also been suggested ${ }^{23}$. A third possibility is that some regions experience both lower sampling effort and greater declines, such that poor data correlates with factors linked to vulnerability, like lower national wealth or conservation investment. Understanding why small time-series contain so many extreme declines is particularly important given that studies that did not find widespread declines often excluded short time-series ${ }^{\text {e.g., }}$,10,12, potentially reconciling divergent findings among studies.

Once extreme clusters were statistically separated, no global trend remained across typical populations (i.e. primary clusters; $98.6 \%$ of populations). However, aggregating systems into one global trend hid important variation. Three systems, all in the Indo-Pacific, showed widespread vertebrate declines across typical populations. Moreover, among typical populations smaller species may be faring worse than larger ones. While tentative given lower sample sizes and high uncertainty, this trend is contrary to common conservation assumptions and so merits additional research.

Our results emphasize an important point: biodiversity trends within and across regions and taxa are highly disparate. This likely reflects differences in both susceptibility and exposure to anthropogenic environmental change ${ }^{24,25,26}$. Unravelling this variation is imperative to understand where biodiversity is most threatened ${ }^{27}$ and which conservation actions promote stability or recovery. A productive global conversation about conservation requires that both scientists and media pay more attention to variation and resist the temptation of simple summary indices.

Shifting the message from ubiquitous catastrophe to foci of concern, also touches on human psychology. Continual negative and guilt-ridden messaging can cause despair, denial and inaction ${ }^{28,29}$. If everything is declining everywhere, despite the expansion of conservation measures in recent decades, it would be easy to lose hope. Our results identify regions that need urgent action to ameliorate widespread biodiversity declines, but also many systems that appear to be generally stable or improving, and thus reason to hope that our actions can make a difference.

\section{References}

1. IUCN. The IUCN Red List of Threatened Species. Version 2019-3. <http://www.iucnredlist.org>. (2019).

2. WWF. Living Planet Report 2018: Aiming higher (eds. Grooten N \& Almond REA). WWF, Gland, Switzerland. (2018). 
3. Rosenberg, K. V et al. Decline of the North American avifauna. Science 366, $120-124$ (2019).

4. Sánchez-Bayo, F. \& Wyckhuys, K. A. G. Worldwide decline of the entomofauna: A review of its drivers. Biol. Conserv. 232 8-27 (2019).

5. Ceballos, G., Ehrlich, P. R. \& Dirzo, R. Biological annihilation via the ongoing sixth mass extinction signaled by vertebrate population losses and declines. Proc. Natl. Acad. Sci. U. S. A. 114, E6089-E6096 (2017).

6. Willig, M. R. et al. Populations are not declining and food webs are not collapsing at the Luquillo Experimental Forest. Proc. Natl. Acad. Sci. U. S. A. 116, 12143-12144 (2019).

7. Daskalova, G. N., Myers-Smith, I. H. \& Godlee, J. L. All is not decline across global vertebrate populations. Preprint at https://www.biorxiv.org/content/10.1101/272898v5. (2018).

8. Dornelas, M. et al. A balance of winners and losers in the Anthropocene. Ecol. Lett. 22, 847-854 (2019).

9. Vellend, M. et al. Global meta-analysis reveals no net change in local-scale plant biodiversity over time. Proc. Natl. Acad. Sci. U. S. A. 110, 19456-19459 (2013).

10. Dornelas, M. et al. Assemblage time series reveal biodiversity change but not systematic loss. Science 344, 296-299 (2014).

11. Gonzalez, A. et al. Estimating local biodiversity change: A critique of papers claiming no net loss of local diversity. Ecology 97, 1949-1960 (2016).

12. Leung, B., Greenberg, D. A. \& Green, D. M. Trends in mean growth and stability in temperate vertebrate populations. Divers. Distrib. 23, 1372-1380 (2017).

13. McGill, B. J., Dornelas, M., Gotelli, N. J. \& Magurran, A. E. Fifteen forms of biodiversity trend in the anthropocene. Trends Ecol. Evol. 30, 104-113 (2015).

14. Anderson, S. C., Branch, T. A., Cooper, A. B. \& Dulvy, N. K. Black-swan events in animal populations. Proc. Natl. Acad. Sci. U. S. A. 114, 3252-3257 (2017).

15. Carrington, D. Humanity has wiped out $60 \%$ of animal populations since 1970 , report finds. The Guardian. <https://www.theguardian.com/environment/2018/oct/30/humanitywiped-out-animals-since-1970-major-report-finds>. (2018)

16. Chung, E. $60 \%$ of world's wildlife has been wiped out since 1970 . <https://www.cbc.ca/news/technology/living-plant-wwf-2018-1.4882819>. CBC News. (2018)

17. Duncan, R. P., Boyer, A. G. \& Blackburn, T. M. Magnitude and variation of prehistoric bird extinctions in the Pacific. Proc. Natl. Acad. Sci. U. S. A. 110, 6436-6441 (2013).

18. Estes, J.A. et al. Trophic downgrading of planet Earth. Science 333, 301-306 (2011).

19. Connors, B. M., Cooper, A. B., Peterman, R. M. \& Dulvy, N. K. The false classification of extinction risk in noisy environments. Proc. R. Soc. B Biol. Sci. 281, 20132935 (2014).

20. Hanks, E. M., Hooten, M. B., Baker, F. A. Reconciling multiple data sources to improve accuracy of large-scale prediction of forest disease incidence. Ecol. Appl. 21, 11731188 (2011).

21. LPI. Living Planet Index database. <www.livingplanetindex.org/>. (2016).

22. Youngflesh, C. \& Lynch, H. J. Black-swan events: Population crashes or temporary emigration? Proc. Natl. Acad. Sci. U. S. A. 114, E8953-E8954 (2017).

23. Fournier, A. M. V., White, E. R. \& Heard, S. B. Site-selection bias and apparent population declines in long-term studies. Conserv. Biol. 33, 1370-1379 (2019). 
24. Newbold, T. et al. Ecological traits affect the response of tropical forest bird species to land-use intensity. Proc. R. Soc. B Biol. Sci. 280, (2013).

25. Venter, O. et al. Sixteen years of change in the global terrestrial human footprint and implications for biodiversity conservation. Nat. Commun. 7, 1-11 (2016).

26. Allan, J. R. et al. Hotspots of human impact on threatened terrestrial vertebrates. PLoS Biol. 17, (2019).

27. Blowes, S. A. et al. The geography of biodiversity change in marine and terrestrial assemblages. Science 366, 339-345 (2019).

28. O'Neill, S. \& Nicholson-Cole, S. "Fear won't do it" Visual and iconic representations. Sci. Commun. 30, 355-379 (2009). Res. 63, 140-146 (2010). 
278 Figure 1. Stylized patterns of system-wide growth rates. Similar geometric mean population growth rates $\left[\log \left(N_{t+1} / N_{t}\right)\right]$ can reflect contrasting systems. As a "null" model, systems can be stable (log growth rates centered at zero, middle panel). Deviations can occur in multiple ways. System can have most populations substantially declining ("Catastrophic" Hypothesis, top panel) or can have multiple clusters with the majority of populations showing a distribution of growth rates centered around zero, but a small cluster of populations experiencing extreme declines ("Clustered" Hypothesis, top-middle panel). Each has the same metric of mean decline (vertical red line $=1.5 \%$ annual decline, corresponding to a $50 \%$ loss over 50 years), even though most populations in the top-middle panel are stable. The converse can also happen, with a small cluster of extremely increasing populations, but otherwise a stable distribution (bottommiddle panel), or most populations increasing (bottom panel) (vertical blue line $=1.5 \%$ annual increase, corresponding to a doubling over 50 years).

Figure 2. Effect of extreme populations on global growth index. Removing a small fraction of extreme populations strongly influences the geometric growth index, using the LPI dataset. Each line represents a different number of populations removed, ranging from no removals (red line: keeping all 14,700 populations, showing a $>50 \%$ mean decline), to removing 356 populations (yellow line: $<2.4 \%$ of populations removed switches the global trend from negative to positive). A geometric growth index of one indicates no change (dashed horizontal black line).

Figure 3. Population trends by taxonomic groups and realms. Top panel shows the terrestrial realm, Middle Panel shows the freshwater realm, and bottom Panel shows the marine realm. Red and blue asterisks denote occurrence of extreme declining clusters (16 systems) and increasing clusters (8 systems), respectively. Distributions show the primary cluster in each system $($ Red $=$ significant declines, Blue $=$ significant increases, Orange $=$ strong non-significant declines, Green = strong non-significant increases, Yellow = weak changes).

Figure 4. Effect of time series size. Number of data points in time series versus the mean log of the geometric mean growth rate.

Figure 5. Populations in the primary clusters across all systems, after removal of extreme clusters. The primary cluster of each system is unimodal, but because systems are experiencing decline (or growth) heterogeneously, plotting distributions across systems shows multimodality. Histograms show significantly declining systems (red), strongly but not significantly declining systems (orange), and weak changes or increases (yellow). Vertical lines show thresholds for strongly declining $(-0.015)$ and strongly increasing $(+0.015)$ growth rates, corresponding to $\sim 50 \%$ loss or a doubling (over 50 years), respectively. Distributions of primary clusters were calculated based on the mean and standard deviations from the hierarchical model, and using the system specific weights to adjust for species richness. 
The publically available LPI data includes 15241 vertebrate populations from 3510 species $^{21}$.

Sensitivity of the "Geometric" indices to extreme population trends country-wide aggregate, we excluded the country-wide aggregate (537 entries), yielding 14700 populations. LPI groups species into 57 systems defined by a combination of habitat domain (terrestrial, freshwater, marine), biogeographic realm (terrestrial/freshwater realms = Afrotropical, Nearctic, Neotropical, Palearctic, Indo-Pacific; marine = Arctic, Atlantic north temperate, Atlantic tropical/sub-tropical, Pacific north temperate, Indo-Pacific tropical/subtropical, South-temperate/Antarctic), and taxonomic grouping (Fish=Actinopterygii, Elasmobranchii, Holocephali, Myxini, Chondrichthyes, Sarcopterygii, Cephalaspidomorphi; Birds=Aves, Mammals=Mammalia, Herps = Amphibia, Reptilia) (Extended data Figure E5-8).

To analyze the effect of body size, we obtained information on each taxonomic group. Given the diversity of vertebrate groups in this data set, and the different conventions across groups, we used different measures of body size for each taxonomic Class based on data availability. For birds $(N=1397)$, mammals $(N=534)$, and reptiles (Squamata, $N=132$; Testudines, $N=44$; and Crocodylia, $N=16$ ) we used estimates of species' mass, in grams, collated in an extensive comparative data set ${ }^{30}$. When mass data were missing for a species $(N=14$ birds; $N=1$ mammal; $N=25$ reptiles), we estimated body mass as the geometric mean of available mass estimates for species in that genus. For fishes (Chondrichthyes, Osteichthyes, Agnatha; $N=$ 1211), estimates of mass were scarce for most species, so we instead used estimates of total length or standard length, in centimetres, both of which were extracted from FishBase ${ }^{31}$ using the rfishbase $\mathrm{R}$ package ${ }^{32}$. These length estimates are an imperfect proxy for size (in terms of mass) given the variability in body plans across groups, but given the large amount of variation across these groups it suffices as a way to broadly categorize species into distinct size classes. For amphibians, we used estimates of snout-vent length ( $S V L$, in $\mathrm{mm}$ ) as our proxy for body size, as this is the most widely available metric of size across species. Data on SVL for amphibian species $(N=175)$ was extracted from a comprehensive ecological trait dataset: AmphiBio ${ }^{33}$.

The LPI analysis was based on a geometric mean approach, calculated by summing across logged growth rates ${ }^{34}$. We recreated the geometric-mean based analyses (see Supplementary Information 1a for full details and model formulation), and examined the sensitivity of the global estimate to extreme populations. We ordered populations and sequentially removed the largest observed decline, determining the effect of each removal on the global estimate of biodiversity loss. Low sensitivity would indicate that many/most populations are declining, supporting the Catastrophic decline hypothesis. High sensitivity, i.e. if removal of relatively few populations switched the strongly negative global trend to neutral or positive, would support the Clustered 
declines hypothesis. For balance, we also examined sensitivity to sequential removal of the greatest increasing populations.

We developed an approach to separate extreme population clusters whose growth or decline statistically deviated from typical population trends, such that a small number of extreme populations would no longer mask trends of the majority of populations (Fig 1). While some summarization is needed to understand global trends, heterogeneous growth rates and potentially multimodal distributions could be expected, given multiple stressors with diverse effects, and differences in species vulnerabilities. We used a Bayesian Hierarchical Mixture (BHM) model as our statistical architecture, as it has several desirable properties: 1) can represent the "null" model and assess deviations from it; 2) allows testing for both negative and positive extremes (sometimes both existed in the same system); 3 ) quantifies the magnitude and proportion of those extremes; 4) provides a coherent way to separate extreme populations from the majority of populations (the primary cluster), enabling tests of the Clustered and Catastrophic declines hypotheses; 5 ) provides a measure of uncertainty as a direct outcome of analysis (via the posterior distribution); and 6) accounts for population fluctuations and adjusts for the number of data points in time-series.

First, we specify the "null" model. Even in a system with no overall trend, we expect stochastic fluctuations in population size. We also expect some populations to be increasing or decreasing during any time interval, given complex, real-world ecological dynamics. Thus, the null model should include among-population heterogeneity, and therefore consists of a distribution of growth rates (Fig 1 middle panel). Statistical deviations from this null model could be caused by a shift in the overall distribution, whereby a system-wide mean growth $<0$ (i.e., decline) could indicate risk to the entire system, and would support the Catastrophic declines hypothesis (Fig 1 top panel). Alternatively, statistical deviation from the null model could be caused by a few populations experiencing extreme declines, consistent with the Clustered declines hypothesis (Fig 1 mid-top panel).

To specify our model, we begin with a standard Bayesian hierarchical formulation (i.e., it does not yet contain mixtures of distributions). We define $\theta, \tau$ as the system-wide mean and variance, respectively, of log-growth rates across all populations in the system (i.e., hyperparameters in Bayesian terminology). $\theta, \tau$ determine the distribution of log population trends $\left(\mu_{i}\right)$, and define the properties of the overall system. However, within population dynamics are also occurring, and the log growth rate for population $i$ at time $t$ are modelled as a population trend $\left(\mu_{i}\right)$ and within population fluctuations $(\sigma)$ (See Supplementary Information $1 \mathrm{~b}$ for full details and model formulation).

Using a standard Bayesian hierarchical model, we can test the Catastrophe hypothesis by determining the probability that the system-wide mean $\theta$ is $<0$. Testing the Cluster hypothesis however requires a mixture model to assess the evidence for the occurrence of clusters. Thus, we define $\mathrm{K}$ is the number of clusters in the mixture, $f_{k}$ is the fraction of populations in the $k$ th cluster, and $\theta, \tau$ and $\boldsymbol{f}$ denote the vectors of parameters for the $K$ clusters. 
To test the Clustered hypothesis we modeled three clusters: a primary cluster, corresponding to the typical trend; a negative extreme cluster; and a positive extreme cluster (Figure 1). Although our main interest was in the mechanisms behind apparent global population declines (i.e. Catastrophic vs. Clustered hypotheses), we also assayed positive extreme clusters so that analyses were not biased to find only negative population trends. We considered four cluster combinations: 1) a single distribution; 2) a primary distribution and a negative extreme distribution; 3) a primary distribution and a positive extreme distribution; or 4) a primary distribution and both positive and negative extreme distributions (Figure 1). For referencing purposes, we denote $k=1$ as the primary cluster, $k=2$ as the negative extreme cluster, and $k=3$ as the positive extreme cluster. Note that reality need not be bi(or tri)-modal, but exploring generalities in trends necessitates some aggregation. Nonetheless, the extreme clusters identified by the mixture model could contain multiple extreme modes in the data (or even result from a skewed distribution). With any of these deviations, model selection (see below) would still choose the mixture model as explaining the data better than a single normal distribution. (See Supplementary Information 1c for full details and model formulation).

We used the (lowest) Deviance Information Criteria (DIC) value to select the mixture model with the strongest statistical evidence ${ }^{35}$. The Catastrophic declines hypothesis would be supported by a mean decline of the primary population cluster $\left(\theta_{1}<0\right.$ and credible intervals did not overlap zero), and would be particularly severe if the mean $\theta_{1}$ was also strongly negative (e.g., $\theta_{1}=-0.015$ would correspond to $>50 \%$ loss over 50 years). The Clustered declines hypothesis would be supported if the DIC selected a mixture with a negative extreme cluster (combinations 2 or 4 above). Note that the Catastrophic and Clustered declines hypotheses are not mutually exclusive, as a system could have both a negative extreme cluster and declining primary cluster. A large fraction of populations in the negative extreme cluster $\left(f_{2}\right)$ could also be interpreted as widespread Catastrophic declines, but this did not occur in our results. Although our hypotheses focus on understanding declining trends, our model will also detect increases in abundances.

To estimate model parameters we used Bayesian analyses and the Markov Chain Monte Carlo algorithm, which simultaneously estimated uncertainty. For each Bayesian analysis, we ran 3 chains, each with 10000 iterations (3000 used for warm-up). Convergence was determined using Rhat $\approx 1$. Values for all parameters across all systems ranged from $(0.999<$ Rhat $<$ 1.005). Bayesian analyses were conducted using the STAN language ${ }^{36}$, and processed and analyzed in $\mathrm{R}^{37}$.

Additionally, we explored the theoretical behavior of each model, including the the geometric mean model, in the presence of clustered declines (see Supplementary Information 1d, 2a), and our Catastrophic and Clustered declines approach given our selection of priors, application of constraints, and other modeling choices; these simulation analyses showed that our approach yielded appropriate theoretic behavior (see Supplementary information 1e, 2b, Extended data Figure E1). Finally, we conducted sensitivity analyses and showed that results were robust to modelling choices (see Supplementary Information 2c, Table S1, Extended data Figure E2). 
450 Application of the Catastrophe and Clusters approach to LPI data

451

452

453

454

455

456

457

458

459

460

461

462

463

464

465

466

467

468

469

470

471

472

473

474

475

476

477

478

479

480

481

482

483

484

485

486

487

488

489

490

491

492

We tested for extreme clusters in each of LPI's 57 domain-realm-taxon systems, by choosing the mixture model with the lowest DIC value. We also examined the number of populations in each cluster, as a fraction of the total number of populations, scaled using the LPI systemspecific weightings $^{38}$ (see Supplementary Information $1 \mathrm{f}$ for more details).

Next, we examined evidence for the Catastrophic declines hypothesis in each system by assaying for negative mean growth rates in the primary cluster $\left(\theta_{1}\right)$. We defined "high certainty" of decline (or increase) as $95 \%$ credible intervals not overlapping zero, and "strong" decline as $\theta_{1}<-0.015$, corresponding to a $\sim 50 \%$ decline if it persisted for 50 years $\left(\theta_{1}>0.015\right.$ was used for a strong positive relations, corresponding to a doubling over 50 years).

We assessed the effect of small time-series on both extreme clusters and trends in primary clusters, by omitting all data with fewer than 10 points, as has often been done in other studies $^{\text {e.g., } 12}$. These small time-series accounted for $52 \%$ of the population estimates $(7110$ populations remained in the analysis).

Finally, we examined whether trends differed between large versus small-bodied animals. Within each Class (but with Agnatha lumped with Osteichthyes), we scaled body size as standard deviations on the natural log-scale - thereby creating an index of relative species size within a taxonomic group. In two cases, we separated out different groups within a Class that had relatively distinct body plans that would influence this size scaling. We scaled size within the Superorder Batoidea (Rajiformes, Myliobatiformes, and Torpediniformes) and separately scaled size for the rest of the Chondrichthyans (Selachimorpha and Holocephali). For the amphibians, we separated out the Orders Caudata and Anura and scaled size within each of these groups. For each taxonomic group we scaled body size and separated species into larger-than-average (henceforth 'larger') versus smaller-than-average (henceforth 'smaller') species. This yielded 9596 populations from 1765 larger species, and 5103 populations from 1745 smaller species. We then reran the BHM model for larger animals and again for smaller animals. Body sizes were divided unevenly among habitat domains and realms; 12 domainrealm-taxon systems contained $\leq 1$ smaller species so were excluded from the small-animal model.

\section{Methods References}

30. Myhrvold, N. P., Baldridge, E., Chan, B., Sivam, D., Freeman, D. L., Ernest, S. K. M., An amniote life-history database to perform comparative analyses with birds, mammals, and reptiles. Ecology 96, 3109 (2015).

31. Froese, R. \& Pauly, D. FishBase. World Wide Web electronic publication. version (12/2019) (2019). Available at: www.fishbase.org.

32. Boettiger, C., Lang, D. T. \& Wainwright, P. C. rfishbase: exploring, manipulating and visualizing FishBase data from R. J. Fish Biol. 81, 2030-2039 (2012). 
33. Oliveira, B. F., São-Pedro, V. A., Santos-Barrera, G., Penone, C. \& Costa, G. C. AmphiBIO, a global database for amphibian ecological traits. Sci. Data 4, 170123 (2017).

34. Collen, B. et al. Monitoring Change in Vertebrate Abundance: the Living Planet Index. Conserv. Biol. 23, 317-327 (2009).

35. Gelman, A., Hwang, J., Vehtari, A. Understanding predictive information criteria for Bayesian models. Statistics and computing 24, 997-1016 (2014).

36. Carpenter, B. et al. Stan: A probabilistic programming language. J. Stat. Softw. 76, (2017).

37. R Core Team. R: A language and environment for statistical computing. R Foundation for Statistical Computing, Vienna, Austria. <http://www.R-project.org/>. (2016).

38. McRae, L., Deinet, S. \& Freeman, R. The diversity-weighted living planet index: Controlling for taxonomic bias in a global biodiversity indicator. PLoS One 12, 1-20 (2017).

\section{Acknowledgements}

We thank E. Hudgins, D. Nguyen, S. Varadarajan, A. Jones for helpful discussions, helpful comments from four anonymous reviewers, and help to create figures from $\mathrm{S}$. Varadarajan and F. Moyes. This work was supported by a Natural Sciences and Engineering Research Council (NSERC) Discovery grant to BL.

\section{Author contributions}

Authors are listed in order of their contributions. BL formulated the BHM model, conducted analyses, and wrote the majority of the ms. AH discussed and clarified the ideas, and also played a central role in writing. DG discussed and clarified the ideas, synthesized the data, and contributed to writing. BM discussed and clarified the ideas, and commented on the ms. MD discussed and clarified the ideas, commented on and improved presentation of the ms. RF discussed and clarified the ideas, and provided insight into the LPI data and analyses.

There are no competing financial interests.

Statement of data availability:

Data can be obtained from the Living Planet Index database. <www.livingplanetindex.org/>. (2016), AmphiBio database from https://figshare.com/articles/Oliveira_et_al_AmphiBIO_v1/4644424>, fishbase database $<w w w$.fishbase.org>, and lifehistory traits from $<$ https://doi.org/10.6084/m9.figshare.c.3308127.v1>

\section{Statement of code availability:}

Code for the BHM model is available at: https://doi.org/10.5281/zenodo.3901586

Supplementary information is available for this paper.

Correspondence and requests for materials should be addressed to brian.leung2@mcgill.ca 
Figure E1. p-p plots showing that the posterior distributions for each estimated parameter are unbiased, and largely follow a 1:1 line for each hyper parameter $(\sigma, \tau)$ as well as the fraction in each cluster $\left(f_{1}, f_{2}=1-f_{1}\right)$. The $1: 1$ line is the theoretic expectation, indicating that the true parameter value to fall below the 0.01 quantile $1 \%$ of the time, 0.02 quantile $2 \%$ of the time, etc.

Figure E2. The trends of the primary clusters $\left(\theta_{1}\right)$, for the main analysis ( $\mathrm{x}$-axis) versus the $N=0$ was observed (bottom panel).

Figure E3. Each point represents a trend estimate for the primary cluster of a system, with the full dataset ( $x$-axis) versus data excluding time series with $<10$ points (y-axis). The red dot was Freshwater Indo-Pacific Mammals, which was reduced from 22 populations (full) to 2 populations (only data $>=10$ points).

Figure E4. Mean trends of primary clusters across systems calculated using the Bayesian Hierarchical Mixture Model. Top) all species (14,700 populations). Middle) only large species (9596 populations). Bottom) only small species (5103 populations). The small species appear to be declining more than large species, although this needs to be interpreted with caution, as most primary distributions did not significantly deviate from zero for small species.

Figure E5. Histograms of observed growth rates and output of the Bayesian Hierarchical Mixture model for systems 1-16 (blue line - primary cluster, red line - extreme cluster(s) from model). Grey vertical lines show the range of observed values. In comparing model output to data: 1) the variation of the BHM primary cluster (blue line) is much lower than the raw data, because the BHM separates variation in among population trends from variation due to within population fluctuations. 2) The BHM model identifies evidence for extreme clusters in both (e.g., Terrestrial Indo-Pacific birds) or only one direction (e.g., Terrestrial Neotropical mammals), but not other apparent clusters (e.g., Terrestrial Indo-Pacific herps). The BHM integrates the magnitude of within population fluctuations, time-series sizes, number of populations, amongpopulation variance, and the magnitude and frequency of the extreme populations in determining whether additional (extreme) clusters are needed to account for the observations.

Figure E6. Histograms of observed growth rates and output of the Bayesian Hierarchical Mixture model for each system 17-32 (blue line - primary cluster, red line - extreme cluster(s) from model). Grey vertical lines show the range of observed values.

Figure E7. Histograms of observed growth rates and output of the Bayesian Hierarchical Mixture model for each system 33-48 (blue line - primary cluster, red line - extreme cluster(s) from model). Grey vertical lines show the range of observed values.

Figure E8. Histograms of observed growth rates and output of the Bayesian Hierarchical Mixture model for each system 49-57 (blue line - primary cluster, red line - extreme cluster(s) from model). Grey vertical lines show the range of observed values. 